\title{
Weakly Regular Subdivisions
}

\author{
Lionel Pournin
}

Received: 23 March 2010 / Revised: 28 June 2011 / Accepted: 26 July 2011 /

Published online: 17 August 2011

(C) Springer Science+Business Media, LLC 2011

\begin{abstract}
It is shown that 2-dimensional subdivisions can be made regular by moving their vertices within parallel 1-dimensional spaces. As a consequence, any 2-dimensional subdivision is projected from the boundary complex of a 4-polytope.
\end{abstract}

Keywords Polyhedral subdivisions $\cdot$ Triangulations $\cdot k$-Regularity $\cdot$ Weak regularity $\cdot$ Steinitz's theorem $\cdot$ Flip-graph connectivity

\section{Introduction}

Let $\mathcal{A}$ be a $d$-dimensional point configuration, that is, a finite subset of $\mathbb{R}^{d}$ whose affine hull has full dimension. A polyhedral subdivision of $\mathcal{A}$ is a collection of subsets of $\mathcal{A}$ whose convex hulls form a polyhedral complex that covers $\operatorname{conv}(\mathcal{A})$. Some of the polyhedral subdivisions of $\mathcal{A}$ can be obtained by projecting the lower facets of a $(d+1)$-dimensional polytope. Such polyhedral subdivisions are called regular and play an important role in many fields of engineering and mathematics. Recently, the author has generalized the notion of regularity using more general polytope projections [14]: A subdivision of a $d$-dimensional point configuration is $k$-regular if it is projected from the boundary complex of a polytope $p \subset \mathbb{R}^{d+k}$. The usual notion of regularity then becomes 1-regularity.

Now consider transforming a subdivision $S$ of $\mathcal{A}$ by moving its vertices while preserving its topological structure. Such transformations are used in triangulationbased algorithms designed for various applications (see [6, 12, 13, 22]). Following

L. Pournin ( $\varangle)$

Mathematics Institute, Ecole Polytechnique Fédérale de Lausanne, 1015 Lausanne, Switzerland e-mail: lionel.pournin@epfl.ch

Present address:

L. Pournin

EFREI, 30-32 avenue de la République, 94800 Villejuif, France

e-mail: lionel.pournin@efrei.fr 
the terminology introduced by Lee [8, 9], a subdivision $S$ is weakly regular if a transformation of this kind results in a regular subdivision of some point configuration. A formal definition of weak regularity is given in Sect. 2.

This paper sheds some light on the link between weakly regular subdivisions and $k$-regularity. It is proven in Sect. 2 that if a subdivision can be transformed into a regular subdivision by moving its vertices within parallel $k$-dimensional affine spaces, then this subdivision is $(k+1)$-regular. In Sect. 3, the case of 2-dimensional subdivisions is addressed. A well known consequence of Steinitz's theorem [20] is that all the subdivisions of 2-dimensional point configurations are weakly regular [1]. It is shown in Sect. 3 that all the subdivisions of any 2-dimensional point configuration can be made regular by moving their vertices along parallel 1-dimensional affine spaces. This implies that all the subdivisions of 2-dimensional point configurations are 2-regular, which settles the 2-dimensional case of a conjecture formulated in [14]. In the last section, it is shown how to regularize a 2-dimensional subdivision by explicitly performing the construction described in Sect. 3 .

\section{Weak Regularity and $k$-Regular Subdivisions}

This section begins with a formal definition of $k$-regularity (see also [14]). A pair $(p, \pi)$ is a polytope projection if $p \subset \mathbb{R}^{n}$ is a polytope and $\pi: \mathbb{R}^{n} \rightarrow \mathbb{R}^{d}$ is an affine map whose restriction to the vertex set of $p$ is injective. Let $(p, \pi)$ be a polytope projection and denote by $\mathcal{V}$ the vertex set of $p$. Let $\phi$ be the inverse of the restriction of $\pi$ to $\mathcal{V}$ (i.e. $\phi$ is the unique map from $\pi(\mathcal{V})$ onto $\mathcal{V}$ so that $\pi \circ \phi$ is the identity). According to the definition found in [3,4], a polyhedral subdivision $S$ is induced by $\pi$ from $p$ if it is a subdivision of $\pi(\mathcal{V})$ so that for all $s \in S$, the convex hull of $\phi(s)$ is a face of $p$. The set of all polyhedral subdivisions induced by $\pi$ from $p$ will be denoted by $\omega(p, \pi)$ in the following of this paper. Note that $\omega(p, \pi)$ can be given the structure of a poset using the refinement relation (see $[3,4])$. The following definition is found in [14]:

Definition 1 A subdivision $S$ is called $k$-regular if there exists a polytope projection $(p, \pi)$ so that $S \in \omega(p, \pi)$ and $\operatorname{dim}(p)-\operatorname{dim}(\pi(p)) \leq k$.

In other words a polyhedral subdivision of a $d$-dimensional point configuration is $k$-regular if it is projected from the boundary complex of a polytope $p \in \mathbb{R}^{d+k}$. According to Proposition 1 in [14], 1-regularity coincides with the usual notion of regularity. Note that regularity is more commonly defined using height functions that lift a $d$-dimensional point configuration to the vertex set of a polytope in $\mathbb{R}^{d+1}$. In the sequel, $v(S)$ will denote the vertex set of a polyhedral subdivision $S$ and $\delta(S)$ the smallest integer $k$ such that $S$ is $k$-regular. A formal definition of weak regularity is now given:

Definition 2 Let $S$ be a subdivision. An injection $\psi: v(S) \rightarrow \mathbb{R}^{d}$ regularizes $S$ if $\psi(S)$ is a regular subdivision of $\psi(v(S))$. 
The set of all injections that regularize a subdivision $S$ will be called $\mathcal{R}(S)$ and $S$ will be called weakly regular if $\mathcal{R}(S) \neq \emptyset$. In other words, a subdivision is weakly regular if, through some motion of its vertices, it can be transformed into a regular subdivision. For any $\psi \in \mathcal{R}(S)$, denote by $\varepsilon(\psi)$ the affine dimension of $\{a-\psi(a)$ : $a \in v(S)\}$ and by $\varepsilon(S)$ minimum of $\varepsilon$ over $\mathcal{R}(S)$; by convention, $\varepsilon(S)=+\infty$ if $\mathcal{R}(S)=\emptyset$.

Note that any subdivision is $k$-regular for some (large enough) integer $k$. Indeed, any subdivision of a $d$-dimensional configuration $\mathcal{A}$ of $n$ points is necessarily ( $n-$ $d-1)$-regular as any subset of $\mathcal{A}$ can be projected from the boundary complex of a $(n-1)$-dimensional simplex. This provides a bound on $\delta(S)$ which depends on the size of $v(S)$. The following theorem gives a better bound on $\delta(S)$ in the case $S$ is weakly regular. Note that this bound only depends on $\varepsilon(S)$ and not on the cardinality of $v(S)$ :

Theorem 1 For any polyhedral subdivision $S, \delta(S) \leq \varepsilon(S)+1$.

Proof Let $d$ be the affine dimension of $v(S)$. Assume without loss of generality that $v(S) \subset \mathbb{R}^{d}$. Let $\psi$ be a map that regularizes $S$ and satisfies $\varepsilon(\psi)=\varepsilon(S)$. As $\psi(S)$ is regular, there exists a polytope projection $(p, \pi)$ so that $p \subset \mathbb{R}^{d+1}$ and for all $s \in$ $\psi(S)$, the convex hull of $\phi(s)$ is a face of $p$, where $\phi$ is the inverse of the restriction of $\pi$ to the vertex set of $p$.

Call $k=\varepsilon(S)+1$. In the remainder of the proof, $\mathbb{R}^{d+1}$ is identified with a subspace of $\mathbb{R}^{d+k}$. Denote by $\kappa$ the orthogonal projection from $\mathbb{R}^{d+k}$ onto $\mathbb{R}^{d+1}$. Since $\operatorname{ker}(\kappa)$ and the affine hull $H$ of $\{a-\psi(a): a \in v(S)\}$ both have dimension $k-1$, there exists an isomorphism $\tau: \operatorname{ker}(\kappa) \rightarrow H$. For all $a \in v(S)$, define

$$
\phi^{\prime}(a)=\phi \circ \psi(a)+\tau^{-1}(a-\psi(a)) .
$$

Note that the restriction of $\kappa$ to $\phi^{\prime}(v(S))$ is an injection into the vertex set of $p$. As a consequence, the convex hull of $\phi^{\prime}(v(S))$ is a polytope $p^{\prime}$ whose vertex set is exactly $\phi^{\prime}(v(S))$. Denote by $\iota$ is the orthogonal projection from $\mathbb{R}^{d+k}$ onto $\operatorname{ker}(\kappa)$ and by $\pi^{\prime}$ the affine map $\pi \circ \kappa+\tau \circ \iota$. According to this construction, the restriction of $\pi^{\prime}$ to $\phi^{\prime}(v(S))$ is a bijection onto $v(S)$ and $\phi^{\prime}$ is its inverse. The pair $\left(p^{\prime}, \pi^{\prime}\right)$ then is a polytope projection.

Now let $s$ be a face of $S$. There exists an affine map $\chi: \mathbb{R}^{d+1} \rightarrow \mathbb{R}$ so that $\operatorname{conv}(\phi \circ$ $\psi(s))$ is the face of $p$ on which $\chi$ is maximal. It follows from the construction of $p^{\prime}$ that $\operatorname{conv}\left(\phi^{\prime}(s)\right)$ is the subset of $p^{\prime}$ that maximizes the affine map $\chi \circ \kappa$. This proves that $\operatorname{conv}\left(\phi^{\prime}(s)\right)$ is a face of $p^{\prime}$ and that $S$ is induced by $\pi^{\prime}$ from $p^{\prime}$. As $p^{\prime} \subset \mathbb{R}^{d+k}$ the result follows.

Let $\mathcal{A}$ be a $d$-dimensional point configuration and $S$ a subdivision of $\mathcal{A}$. If $S$ is weakly regular then necessarily, $\varepsilon(S) \leq d$. The corollary below therefore immediately follows from Theorem 1:

Corollary 1 Any weakly regular subdivision of a d-dimensional point configuration is $(d+1)$-regular. 
The end of this section focuses on the notion of flip. Let $T$ be a triangulation of some point configuration $\mathcal{A}$, that is, a subdivision of $\mathcal{A}$ whose elements are affinely independent. Under given conditions, it is possible to transform $T$ into another triangulation of $\mathcal{A}$ performing a local operation in $T$ called flip. This operation basically consists in replacing the star of a simplex in $T$ by another triangulation of this star. An exact definition of flips is not required nor given here; the interested reader will find formal definitions in [14, 17-19]. The graph whose vertices are the triangulations of $\mathcal{A}$ and whose edges link two triangulations that can be transformed into one another by a flip is called the flip-graph of $\mathcal{A}$.

For practical applications, flip-graph connectivity, especially in the case of 3-dimensional point configurations, is an important issue as flips are used to build regular triangulations from non-regular ones. It is shown in [14] that the subgraph induced in the flip-graph of $\mathcal{A}$ by 2-regular triangulations is connected. Hence the following result becomes a consequence of Theorem 1 :

Corollary 2 Let $T$ be a triangulation of some d-dimensional point configuration. If $\varepsilon(T) \leq 1$ then $T$ can be flipped to a regular triangulation.

Note that flip-graph connectivity for general 3- and 4-dimensional point configurations remains an open question. For 2-dimensional point configurations, it was shown by Lawson [7] that flip-graphs are always connected. The next section focuses on dimension 2: it is proven that if $S$ is a subdivision of a 2-dimensional point configuration then $\varepsilon(S) \leq 1$. This will have several consequences, among which an alternative proof for the connectivity result in [7].

\section{Two-Dimensional Subdivisions Are Weakly Regular}

Let $S$ be a subdivision of some 2-dimensional point configuration. The set of the interior vertices of $S$ will be denoted by $v_{i}(S)$ and the set of its interior edges by $e_{i}(S)$. For any vertex $a$ of $v(S)$, denote by $l(a, S)$ the set of all vertices of $S$ adjacent to $a$. It will be shown in this section that $\varepsilon(S) \leq 1$. A characterization of regularity originally formulated by Maxwell $[10,11]$ will be used to this end. This characterization links the local convexity or concavity of a 3-dimensional polyhedral surface to the signs of the constraints induced in its edges as they are projected into the plane. Several results have been obtained since then on the topic (see in particular [16, 21]). In this characterization, the sufficient condition for regularity does not require the set of edges in the plane to be the 1-skeleton of a polyhedral complex: edges could a priori cross and cells be non-convex. This is particularly convenient when displacing the points of a polyhedral subdivision. The following result is a direct consequence of Theorem 5.1 in [16]:

Theorem 2 Let $S$ be a subdivision of some 2-dimensional point configuration and $\psi: v(S) \rightarrow \mathbb{R}^{2}$ an injection so that $\psi\left(v(S) \backslash v_{i}(S)\right)$ lies within the relative boundary 
of its convex hull. Let $\tau: e_{i}(S) \rightarrow \mathbb{R}$ be a map so that for all $a \in v_{i}(S)$,

$$
\sum_{b \in l(a, S)} \tau(\{a, b\})(\psi(b)-\psi(a))=0 .
$$

If $\tau$ is positive on all the interior edges of $S$ then there exists a regular subdivision $R$ of $\psi(v(S))$ so that $\psi(S) \subset R$.

The lemma below will be useful in the proof of Theorem 3. It states sufficient conditions under which a square matrix admits an inverse. Similar conditions for matrix non-singularity can be found in [2].

Lemma 1 Let $M$ be a square matrix whose columns sum to a non-zero, non-negative vector. If $\alpha I-M$ is non-negative and $(\alpha I-M)^{k}$ is positive for some $\alpha>0$ and some $k \in \mathbb{N}$ then $M$ admits an inverse.

Proof Call $n$ the number of lines of matrix $M$ and denote $A=I-\alpha^{-1} M$. For any $i \in \mathbb{N}$, denote by $y_{i}$ the vector obtained by summing the columns of $A^{i}$. According to the hypotheses, $A$ is a non-negative matrix and so are its successive powers. Hence $y_{i}$ is non-negative for all $i \in \mathbb{N}$. Moreover, as the columns of $M$ sum to a non-negative vector, then all the coefficients of $y_{1}$ are in $[0,1]$.

Let $i$ be a positive integer. By writing the coefficients of $A^{i+1}$ as scalar products of the rows of $A^{i}$ with the columns of $A$, one finds that $y_{i+1}=A^{i} y_{1}$. In other words, the coefficients of $y_{i+1}$ are the scalar product of the rows of $A^{i}$ with vector $y_{1}$. As both $A^{i}$ and $y_{1}$ are non-negative and as 1 is an upper bound on the coefficient of $y_{1}$, one then obtains $y_{i+1} \leq y_{i}$.

Using this last inequality recursively, one shows in particular that for all $i \in \mathbb{N}$, all the coefficients of $y_{i}$ are in $[0,1]$. According to the hypotheses, $A^{k}$ is a positive matrix. Moreover, as the columns of $M$ sum to a non-zero vector, then at least one coefficient of $y_{1}$ is strictly smaller than 1 . One therefore deduces from the equality $y_{k+1}=A^{k} y_{1}$ that the strict inequality $y_{k+1}<y_{k}$ holds, which proves that every row of matrix $A^{k+1}$ sums to a number in [0, $1[$.

For every vector $x \in \mathbb{R}^{n}$, the inequality $\left\|A^{k+1} x\right\|_{\infty}<\|x\|_{\infty}$ then holds. As a consequence, all the eigenvalues of $A^{k+1}$ have their moduli in [0, 1 [ and so do the eigenvalues of $A$. This proves that the series $\sum_{i \geq 0} A^{i}$ converges, which is equivalent to the non-singularity of $I-A$ (see Lemma 2.1 in Chap. 6 of [2]). As matrix $M$ is equal to $I-A$ up to a non-zero multiplicative coefficient, the lemma is proven.

The following theorem is the main result of this paper. Not only does it show that all the subdivisions of 2-dimensional point configurations are weakly regular but also that it is possible to regularize such subdivisions by moving their vertices along parallel lines.

Theorem 3 If $S$ is a subdivision of a 2-dimensional point configuration then $S$ is weakly regular and $\varepsilon(S) \leq 1$. 
Proof Let $u$ and $v$ be two orthogonal unit vectors in $\mathbb{R}^{2}$ such that all the vertices of $S$ have distinct coordinates along $v$. In a first step, it is shown that there is a map $\left.\tau: e_{i}(S) \rightarrow\right] 0,+\infty\left[\right.$ so that for all $a \in v_{i}(S)$,

$$
\sum_{b \in l(a, S)} \tau(\{a, b\})(b-a) \cdot v=0 .
$$

This will be achieved by inductively building a map $\left.\sigma: v_{i}(S) \rightarrow\right] 0,+\infty[$ that satisfies given properties and subsequently deducing $\tau$ from $\sigma$. For any vertex $a \in v(S)$, call $v^{-}(a, S)$ the set of all vertices $b \in v(S)$ so that $b \cdot v \leq a \cdot v$ and denote:

$$
l^{-}(a, S)=\left\{b \in v^{-}(a, S):\{a, b\} \in e_{i}(S)\right\}
$$

In other words, $l^{-}(a, S)$ is the set of the vertices $b$ of $S$ whose coordinates along $v$ are smaller than $a \cdot v$ and so that $\{a, b\}$ is an interior edge of $S$. Call $n^{+}(a, S)$ the number of the edges $\{a, b\}$ interior to $S$ that satisfy $a \cdot v<b \cdot v$. Now let $a \in v(S)$. It is proven by induction on the cardinality of $v^{-}(a, S)$ that there exists a map $\sigma$ : $\left.v^{-}(a, S) \rightarrow\right] 0,+\infty\left[\right.$ so that for all $x \in v^{-}(a, S) \cap v_{i}(S)$,

$$
n^{+}(x, S) \sigma(x)=\sum_{y \in l^{-}(x, S)} \sigma(y) .
$$

If $v^{-}(a, S)$ only has one element then this element is necessarily $a$ and one defines a positive map on $v^{-}(a, S)$ by choosing an arbitrary positive number for $\sigma(a)$. Notice that, at this stage, (3) does not need to be satisfied as $a \notin v_{i}(S)$. Now assume that $v^{-}(a, S)$ contains at least two vertices. Call $a^{\prime}$ the vertex in $v^{-}(a, S)$ that satisfies $v^{-}(a, S)=v^{-}\left(a^{\prime}, S\right) \cup\{a\}$. By induction there exists a map $\left.\sigma: v^{-}\left(a^{\prime}, S\right) \rightarrow\right] 0,+\infty[$ so that for all $x \in v^{-}\left(a^{\prime}, S\right) \cap v_{i}(S)$, (3) is satisfied. This map can be extended to $v^{-}(a, S)$ by choosing a positive number for $\sigma(a)$ if $a$ is in the boundary of $S$ and,

$$
\sigma(a)=\frac{1}{n^{+}(a, S)} \sum_{y \in l^{-}(a, S)} \sigma(y),
$$

if $a$ is interior to $S$. Doing so is possible as, by induction, $\sigma$ already has been defined on $l^{-}(a, S)$. Observe then that $\sigma(a)>0$ and further that if $a$ is interior to $S,(3)$ is necessarily satisfied for $x=a$, which finishes the induction.

This shows the existence of a positive map $\sigma$ on $v(S)$ so that for every interior vertex $x$ of $S$, (3) is satisfied. Now consider an interior edge of $S$, denote by $a$ and $b$ its vertices with the convention that $a \cdot v<b \cdot v$, and call

$$
\tau(\{a, b\})=\frac{\sigma(a)}{(b-a) \cdot v} .
$$

By (3), this defines a positive map $\tau$ on $e_{i}(S)$ so that (2) is satisfied for every interior vertex $a$ of $S$. In a second step, an injection $\psi: v(S) \rightarrow \mathbb{R}^{2}$ will be found so that (1) holds for all the interior vertices of $S$ with map $\tau$. 
For every vertex $a \in v_{i}(S)$, call $\beta_{a}=\sum_{b \in l(a, S)} \tau(\{a, b\})(b-a) \cdot u$ and consider the following linear equation system with variables $\left(x_{a}\right)_{a \in v_{i}(S)}$ :

$$
\forall a \in v_{i}(S), \quad\left[\sum_{b \in l(a, S)} \tau(\{a, b\})\right] x_{a}-\sum_{\substack{b \in l(a, S) \\ b \in v_{i}(S)}} \tau(\{a, b\}) x_{b}=\beta_{a} .
$$

As a matrix equation, this system reads $M X=B$, where $X$ and $B$ are the vectors with coefficients $x_{a}$ and $\beta_{a}$, respectively, and $M$ is a square matrix whose diagonal coefficients are the sums $\sum_{b \in l(a, S)} \tau(\{a, b\})$ for $a \in v_{i}(S)$ and whose non-diagonal coefficients are either equal to zero or to $-\tau(\{a, b\})$ for some $(a, b) \in v_{i}(S)^{2}$. Hence, the diagonal coefficients of $M$ are positive and its non-diagonal coefficients are nonpositive. Moreover, the sum of the coefficients of matrix $M$ in the row corresponding to a vertex $a \in v_{i}(S)$ is either positive (if $l(a, S) \not \subset v_{i}(S)$ ) or equal to zero (if $l(a, S) \subset$ $\left.v_{i}(S)\right)$. As there necessarily exist vertices in $v_{i}(S)$ adjacent to some boundary vertex of $S$, the columns of matrix $M$ sum to a non-zero, non-negative vector. Call $\alpha$ the largest diagonal coefficient of $M$. The matrix $\alpha I-M$ is non-negative and its nonzero off diagonal coefficients exactly correspond to the edges in the 1-skeleton of $S$. As the 1-skeleton of $S$ is a connected graph, there exists an integer $k \in \mathbb{N}$ so that $(\alpha I-M)^{k}$ is positive. According to Lemma $1, M$ is therefore invertible and system (4) admits a unique solution. Let $\left(x_{a}\right)_{a \in v_{i}(S)}$ be this solution. For all $a \in v_{i}(S)$, define $\psi(a)=a+x_{a} u$ and for all $a \in v(S) \backslash v_{i}(S)$, define $\psi(a)=a$. Using $\psi$ instead of $\left(x_{a}\right)_{a \in v_{i}(S)}$ in the system of (4), it is found that, for all $a \in v_{i}(S)$,

$$
\sum_{b \in l(a, S)} \tau(\{a, b\})(\psi(b)-\psi(a)) \cdot u=0 .
$$

Let $a$ be a vertex of $S$. As $a$ and $\psi(a)$ have the same coordinate along $v$, one obtains from (2) and (5) that (1) holds for vertex $a$. This shows that maps $\tau$ and $\psi$ satisfy the hypotheses of Theorem 2. According to this theorem, $\psi(S)$ is a subset of some regular subdivision $R$ of $\psi(v(S)$ ). As $R$ and $\psi(S)$ are (topologically) 2-dimensional balls with the same boundary, this inclusion implies the equality $\psi(S)=R$. This proves that $\psi \in \mathcal{R}(S)$ and that $S$ is weakly regular. Moreover, the affine hull of $\{a-\psi(a)$ : $a \in v(S)\}$ is either equal to $\{0\}$ or to the 1-dimensional space spanned by vector $u$. As a consequence $\varepsilon(S) \leq 1$.

Observe that the proof of Theorem 3 gives an explicit way to regularize a subdivision $S$ of any 2-dimensional point configuration: first, a positive map $\tau$ on the interior edges of $S$ is built by considering the vertices of $S$ ordered along a given axis. At this stage $\tau$ satisfies (2) for all $a \in v_{i}(S)$. Secondly, the system of (4) is solved, which provides a map $\psi$ that defines motions for the interior vertices of $S$ so that $\psi(S)$ is a regular subdivision of $\psi(v(S))$. This construction will be explicitly carried out on an example in Sect. 4.

Three corollaries of Theorem 3 are now given. Let $S$ be a subdivision of some 2 -dimensional point configuration. As a consequence of Theorems 1 and $3, S$ is 2-regular. In other words, it is projected from the boundary complex of some 4-dimensional polytope. This settles the 2-dimensional case of the conjecture formulated in [14]: 
Corollary 3 All the subdivisions of any 2-dimensional point configuration are 2-regular.

Observe that this corollary is a tight result as there exist 2-dimensional point configurations with non-regular subdivisions (see Sect. 4). The two following corollaries only give alternative proofs to already known results. It is a well known consequence of Steinitz's theorem [20] that every 3-connected planar graph is isomorphic to the 1 -skeleton of a polyhedral subdivision of some 2-dimensional point configuration. Indeed, if $p$ is the polytope built following Steinitz's theorem, such a subdivision can be obtained by centrally projecting the boundary complex of $p$ from a point placed close to one of its 2-dimensional faces. The obtained subdivision would be regular if a linear projection were used instead of a central one. This issue was settled in [1] where it is proven that one can prescribe the faces of $p$ that (linearly) project to the boundary of the subdivision. Theorem 3 provides an alternative proof of this result:

Corollary 4 Every 3-connected planar graph is isomorphic to the 1-skeleton of some regular subdivision of a 2-dimensional point configuration.

Finally, recall that the subgraph induced by 2-regular subdivisions in the flip-graph of a point configuration is connected (Theorem 2 in [14]). Hence, the following result, already obtained by Lawson in [7], gets an alternative proof from the combination of Corollary 3 with [14]:

Corollary 5 The flip-graph of any 2-dimensional point configuration is connected.

\section{Examples and Discussion}

In this section, the construction used in the proof of Theorem 3 is explicitly applied to the non-regular triangulation shown in the left of Fig. 1. Let $d$ and $e$ be two positive numbers. The matrix below gives the coordinates in $\mathbb{R}^{2}$ of six points that collectively form a point configuration $\mathcal{A}$. Those coordinates are expressed in an orthogonal basis $(u, v)$ of $\mathbb{R}^{2}$. Each column of the matrix corresponds to a point whose coordinates along $u$ and $v$ can be, respectively, read in the first and second row:

$$
\mathcal{A}=\left(\begin{array}{cccccc}
-d / 2 & (e-1) / 2 & d & 1+e / 2 & -e-1 / 2 & -d / 2 \\
-\sqrt{3} d / 2 & -\sqrt{3}(e+1) / 2 & 0 & \sqrt{3} e / 2 & \sqrt{3} / 2 & \sqrt{3} d / 2
\end{array}\right) .
$$

Each point in $\mathcal{A}$ will be referred to using the number of its column in the above matrix, and subsets of $\mathcal{A}$ will be denoted by simply enumerating the points they contain. Consider the following subsets of $\mathcal{A}$ :

$$
124,126,134,245,256,345,356 .
$$

If $d>1$ and $e<\left(\sqrt{d^{2}+6 d-3}-d-1\right) / 2$, then these seven subsets of $\mathcal{A}$ are the 2-dimensional faces of a non-regular triangulation $T_{d, e}$ of $\mathcal{A}$. This triangulation is depicted on the left of Fig. 1 for $d=3$ and $e=1 / 3$. One can check that $T_{d, e}$ is 

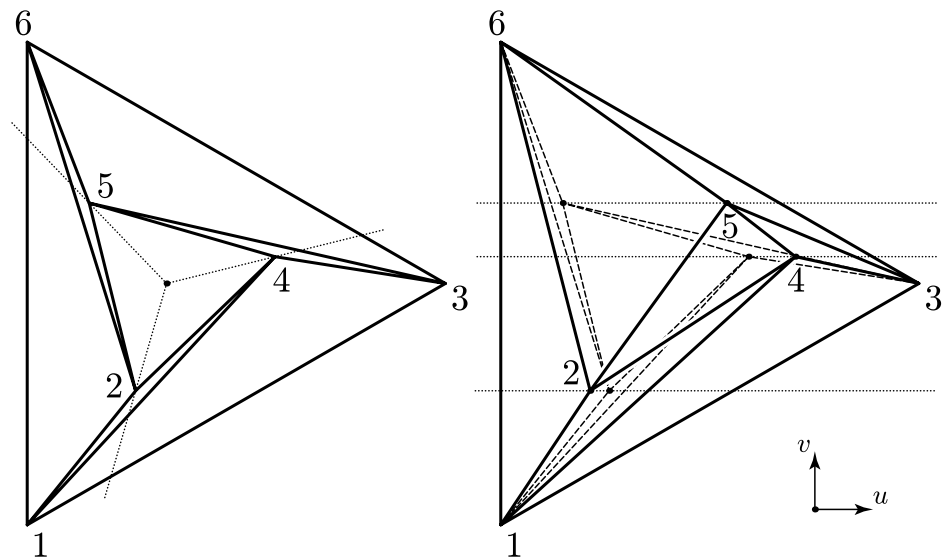

Fig. 1 A non-regular subdivision (left) and its regularization (right) constructed by the proof of Theorem 3

actually non-regular by using the acyclicity criterion described in [5]: edges 14, 26, and 35 form a cycle of overlapping faces with respect to the centroid of triangle 245 . The dotted lines drawn in the left of Fig. 1 help visualize this property. It is proven in [5] that a subdivision with such a cycle is necessarily non-regular.

In the proof of Theorem 3, an orthonormal basis $(u, v)$ is first chosen so that the points in $\mathcal{A}$ have distinct coordinates along $v$. The basis chosen here to express the coordinates of the points in $\mathcal{A}$ already fulfills this requirement. Next, a map $\sigma$ : $\mathcal{A} \rightarrow] 0,+\infty$ [ is built so that (3) is satisfied by every vertex $x$ interior to $T_{d, e}$. Here, following the procedure found in this proof, and choosing $\sqrt{3} / 2$ as the image under $\sigma$ of all the boundary vertices of $T_{d, e}$, one obtains

$$
\sigma(2)=\frac{\sqrt{3}}{6} ; \quad \sigma(4)=\frac{7 \sqrt{3}}{6} ; \quad \sigma(5)=\frac{11 \sqrt{3}}{6} .
$$

A map $\left.\tau: e_{i}\left(T_{d, e}\right) \rightarrow\right] 0,+\infty[$ is then constructed from map $\sigma$ and from the coordinates along $v$ of the vertices of $T_{d, e}$. Here, one finds

$$
\begin{array}{llll}
\tau(12)=1 /(d-e-1) ; & \tau(14)=1 /(d+e) ; & \tau(24)=1 /(3+6 e) ; \\
\tau(25)=1 /(6+3 e) ; & \tau(26)=1 /(3+3 e+3 d) ; & \tau(34)=1 / e \\
\tau(35)=1 ; & \tau(45)=7 /(3-3 e) ; & \tau(56)=11 /(3 d-3) .
\end{array}
$$

The system (4) can then be written using those values for $\tau$ and solved. While this can actually be done formally as a function of $d$ and $e$, the expression of the solution obtained is very complicated and will be omitted here. The exact value of this solution for $d=3$ and $e=1 / 3$ is given instead: $x_{2}=-214 / 1047, x_{4}=176 / 349$, and $x_{5}=$ $1846 / 1047$. By Theorem 3, those quantities are motions along vector $u$ of vertices 2 , 4 , and 5, respectively, so that the resulting triangulation is regular. This triangulation is shown on the right of Fig. 1, where the original triangulation is drawn with dashed lines and the motion of the vertices takes place along the horizontal dotted lines. One can see in particular that edges 14,26 , and 35 no longer form a cycle of overlapping faces after the motion of the vertices. 
A natural question is whether Theorem 3 carries over to higher dimensions. The answer is given by Rudin's triangulation [15]. This triangulation of a 3-dimensional point configuration is non shellable. Non shellability is a topological property and as a consequence, any triangulation obtained by moving the vertices of Rudin's triangulation while preserving its topology will remain non shellable. As regularity implies shellability, Rudin's triangulation cannot be weakly regular. Hence, above dimension 2, subdivisions exist that are not weakly regular. It has been shown in [14], though, that Rudin's triangulation is 2-regular. While it is unlikely that Corollaries 3 and 5 carry over to higher dimensions (see [14]), a subdivision of some 3-dimensional point configuration that is not 2-regular is yet to be found.

Finally, in the perspective of practical applications [6, 12, 13, 22], the key question is whether a subdivision known to be weakly regular can be transformed by flips into a regular subdivision. While this question remains open in dimension 3 , the tools described in this paper contribute to provide insights on possible answers.

Acknowledgement I am grateful to Tom Liebling for his kind and patient help with the writing of this article, and for his friendship.

\section{References}

1. Barnette, D.W.: Projections of 3-polytopes. Isr. J. Math. 8, 304-308 (1970)

2. Berman, A., Plemmons, R.J.: Nonnegative Matrices in the Mathematical Sciences. Classics in Applied Mathematics, vol. 9. SIAM, Philadelphia (1994)

3. Billera, L.J., Sturmfels, B.: Fiber polytopes. Ann. Math. 135, 527-549 (1992)

4. Billera, L.J., Kapranov, M.M., Sturmfels, B.: Cellular strings on polytopes. Proc. Am. Math. Soc. 122, 549-555 (1994)

5. Edelsbrunner, H.: An acyclicity theorem for cell complexes in $d$ dimension. Combinatorica 10, 251260 (1990)

6. Ferrez, J.-A., Liebling, Th.M.: Dynamic triangulations for efficient detection of collisions between spheres with applications in granular media simulations. Philos. Mag., B 82, 905-929 (2002)

7. Lawson, C.L.: Software for $C^{1}$ interpolation. In: Rice, J. (ed.) Mathematical Software III, pp. 161194. Academic Press, New York (1977)

8. Lee, C.W.: Regular triangulations of convex polytopes. In: Gritzmann, P., Sturmfels, B. (eds.) Applied Geometry and Discrete Mathematics-The Victor Klee Festschrift. DIMACS Series in Discrete Mathematics and Theoretical Computer Science, vol. 4, pp. 443-456. American Mathematical Society, Providence (1991)

9. Lee, C.W.: Subdivisions and triangulations of polytopes. In: Goodman, J.E., O'Rourke, J. (eds.) Handbook of Discrete and Computational Geometry, pp. 383-406. CRC Press, Boca Raton (2004)

10. Maxwell, J.C.: On reciprocal figures and diagrams of forces. Philos. Mag. Ser. 7 27, $250-261$ (1864)

11. Maxwell, J.C.: On reciprocal figures frames, and diagrams of forces. Trans. R. Soc. Edinb. 26, 1-40 (1869)

12. Pournin, L., Liebling, Th.M.: Constrained paths in the flip-graph of regular triangulations. Comput. Geom. 37, 134-140 (2007)

13. Pournin, L., Liebling, Th.M.: From spheres to spheropolyhedra: generalized Distinct Element Methodology and algorithm analysis. In: Cook, W., Lovász, L., Vygen, J. (eds.) Research Trends in Combinatorial Optimization, pp. 347-363. Springer, Berlin (2009)

14. Pournin, L.: A result on flip-graph connectivity. Adv. Geom. (in press)

15. Rudin, M.E.: An unshellable triangulation of a tetrahedron. Bull. Am. Math. Soc. 64, 90-91 (1958)

16. Rybnikov, K.: Stresses and liftings of cell-complexes. Discrete Comput. Geom. 21, $481-517$ (1999)

17. Santos, F.: A point set whose space of triangulations is disconnected. J. Am. Math. Soc. 13, 611-637 (2000)

18. Santos, F.: On the refinements of a polyhedral subdivision. Collect. Math. 52, 231-256 (2001) 
19. Santos, F.: Geometric bistellar flips: the setting, the context and a construction. In: Sanz-Sole, M., Soria, J., Varona, J.L., Verdera, J. (eds.) Proceedings of the ICM 2006, vol. III, pp. 931-962. Eur. Math. Soc., Zurich (2006)

20. Steinitz, E.: Polyeder und Raumeinteilungen. Enzykl. Math. Wiss. 3, 1-139 (1922)

21. Whiteley, W.: Motions and stresses of projected polyhedra. Topol. Struct. 7, 13-38 (1982)

22. Xue, X.J., Righetti, F., Telley, H., Liebling, Th.M., Mocellin, A.: The Laguerre model for grain growth in three dimensions. Philos. Mag., B 75, 567-585 (1997) 